\title{
$\bullet$ COVID-19 Treatment Options and Vaccination in India: A Perspect
}

\section{IJCRR}

Section: Healthcare

ISI Impact Factor

(2019-20): 1.628

IC Value (2019): 90.81 SJIF (2020) $=7.893$

\section{Nagur Sharone Grace, Sowmini K*, Veena B, Vadlakonda Sruthi}

Junior Resident, Department of Pharmacology, Osmania Medical College, Hyderabad, Telangana - 500o95, India

\section{ABSTRACT}

The origin of Severe Acute Respiratory Syndrome Coronavirus -2 [SARS CoV-2] from Wuhan city, China advanced to the catastrophic pandemic. There are attempts universally to gear up the potential therapeutic line in the treatment of COVID-19. The current management for COVID-19 is leaning towards the repurposing of drugs based on preceding outbreak data [SARS 20022003, MERS-2012] and structural organization and genomics of the coronavirus. Unfortunately, no specific therapy is available to treat coronavirus infection amidst this emergency. Antibody improvement is a key element for decreasing mortality related to viral diseases. Viral vaccines have to be immunogenic, safe, efficacious and suitable to induce long-lasting immunity. Studies are being expedited right now in the development of a vaccine for coronavirus globally. India with a dense population and enormous resources related to pharmaceuticals can be one of the bulk manufacturers of Coronavirus vaccines. This article focuses on the outline of therapeutic options, vaccine development strategy and highlights the current status of vaccines trials in India.

Key Words: Immunity, India, Repurposed drugs, SARS CoV-2, Status, Vaccine development

\section{INTRODUCTION}

Coronavirus disease (COVID-19) is disrupting infectious disease. An epidemic of acute respiratory syndrome in humans appeared in Wuhan, China in December 2019, was caused by this novel coronavirus (SARS-CoV-2) and is now spread worldwide. ${ }^{1}$ When compared to SARS and MERS, severe acute respiratory syndrome-related coronaviruses (SARS-Co V) spread swiftly. The outbreak was declared as "a public health emergency of international concern" by the WHO on January 30, 2020, and as a pandemic on March 11,2020 , as there is a rapid increment of cases globally. ${ }^{2}$ Fever $(88.5 \%)$, cough $(68.6 \%)$, myalgia or fatigue $(35.8 \%)$, expectoration (28.2\%), and dyspnea $(21.9 \%)$ are the primary clinical symptoms and the minor symptoms are headache or dizziness $(12.1 \%)$, diarrhoea $(4.8 \%)$, nausea and vomiting $(3.9 \%){ }^{3}$ Globally as of $2^{\text {nd }}$ September 2020 , there have been 25,602,665 confirmed cases of COVID-19 reported at WHO, with Indian statistics of 3.85 million cases.

The viral genome of SARS-CoV-2 was rapidly sequenced to enable diagnostic testing, epidemiologic tracking, and the de- velopment of preventive and therapeutic strategies. Though many drugs are being used in COVID 19 treatment including the concept of drug repurposing and few in the clinical pipeline, promising results are not witnessed in narrowing down mortality and morbidity numerical. As of 10 August, search terms for COVID 19 resulted in 2935 studies on clinical trials .gov. There are no FDA (Food and Drug Administration) approved drugs for the treatment of COVID-19. However, there are few drugs in the race in terms of management for COVID-19.

\section{HYDROXYCHLOROQUINE (HCQ) \& CHLORQUINE (CQ)}

CQ was shown to potently inhibit the entry of SARS-Co V-2 into cells by interfering with the glycosylation of its cellular receptor angiotensin-converting enzyme -2 receptor (ACE2). Coronaviruses such as SARS-Co V were shown to be able to enter target cells via a $\mathrm{pH}$-dependent mechanism. CQ alter acidic $\mathrm{pH}$ of the lysosome disfavouring and interfering fusion of the viral and endosomal membranes involved in viral particle uncoating and subsequent release of viral nucleic

\section{Corresponding Author:}

Sowmini K, Junior Resident, Department of Pharmacology, Osmania Medical College, Hyderabad, Telangana - 500095, India.

ISSN: 2231-2196 (Print)

Received: 22.12 .2020
ISSN: 0975-5241 (Online)

Revised: 12.01 .2021
Accepted: 23.01 .2021
Published: 30.03 .2021 
acid into the cytoplasm. CQ can also impair posttranslational modifications of viral proteins through interfering with proteolytic processes and inhibition of glycosylation via specific interactions with sugar-modifying enzymes or glycosyltransferases. CQ works as an anti-inflammatory agent by reducing tumour necrosis factor $(\mathrm{TNF} \alpha)$ release and suppressing TNF receptors on monocytes. ${ }^{4} \mathrm{HCQ}$ and CQ share a common chemical structure \& Mechanism of action. HCQ has lower toxicity compared to CQ. HCQ also has immunosuppressive properties that may help reduce the cytokine storm in severe COVID-19. In terms of adverse effects, though HCQ is safe it may cause QT segment prolongation leading to arrhythmia and myocardial arrest. ${ }^{5} \mathrm{HCQ}+$ azithromycin invitro showed good results but associated with high cardiovascular abnormalities.

\section{Renin Angiotensin system (RAS)}

Harmful and Beneficial effects are the two opposite hypotheses proposed for RAS Inhibitors on the lungs). In the "harmful effect" hypothesis, upregulation of ACE2 expression is done by RAS inhibitors promoting SARS-CoV-2 entry. In the "beneficial effect" hypothesis, RAS inhibitors reduce inflammation and fibrosis in the lung mediated by the Ang II-dependent AT1 receptor. According to a study, RAS inhibitors do not directly inhibit viral replication but pose an indirect antiviral role by regulating immune function and inhibiting inflammatory responses. RAS Inhibitor therapy inhibits IL-6 levels and attenuates the inflammatory response. These findings are consistent with the alleviation of lipopolysaccharide-induced pneumonic injury by RAS Inhibitors. ${ }^{6,7}$

\section{HIV PROTEASE INHIBITORS}

\section{Lopinavir/ritonavir}

3-chymotrypsin-like protease (3CLpro) is the enzyme involved in SARS-CoV-2 replication. In in vitro, Lopinavir/ ritonavir can inhibit the 3CLprotease enzyme which appears to be highly conserved in SARS-CoV-2. Coronavirus-infected enterocytes might be exposed to higher concentrations of lopinavir as the drug is excreted in the gastrointestinal tract. ${ }^{8}$

\section{Darunavir}

Darunavir inhibits the 3-Chymotrypsin Like proenzyme of SARS-CoV-2 and possibly also inhibits the Papain Like proenzyme. The results from an in vitro study and an unpublished randomized controlled trial of 30 patients in China, darunavir did not show activity against SARS-CoV-2. ${ }^{9}$

\section{Ribavirin}

Ribavirin a guanine analogue inhibits viral RNA-dependent RNA polymerase can be used against SARS-Co V for COV-
ID - 19 treatment. However, in vitro activity against SARSCo $\mathrm{V}$ was limited and required high concentrations to inhibit viral replication. ${ }^{8}$

\section{Atazanavir}

Invitro studies against SARS-CoV-2 with atazanavir inhibits the cysteine protease activity of cell lysates containing Major protease. Atazanavir treated human epithelial pulmonary cell line (A549) infected with SARS-CoV-2 displayed reduced viral replication. Besides that SARS-CoV-2 infected human monocytes, on treatment with the Atazanavir/Ritonavir combination lowered IL-6 and TNF-alpha secretion. ${ }^{10}$

\section{ANTIVIRALS}

\section{Oseltamivir}

Oseltamivir targets the neuraminidase distributed on the surface of the influenza virus to inhibit the spread of the influenza virus in the human body. A study in Wuhan reported that no positive outcomes were observed after receiving antiviral treatment with oseltamivir. ${ }^{11}$

\section{Favipiravir}

Approved by Japan for the treatment of avian influenza or novel influenza resistant to neuraminidase inhibitors. Recent in vitro and human studies have repurposed Favipiravir as an experimental agent against enveloped, positive-sense, single-strand RNA virus SARS-CoV-2. Also, the study showed Favipiravir has exerted efficacy in Vero E6 cells infected with SARS-CoV-2 with half-maximal effective concentration implying the high concentration is needed for safe and effective treatment. ${ }^{11}$

\section{Arbidol (Umifenovir)}

Broad-spectrum direct-acting antiviral with a unique mechanism of action targeting the S protein/ACE2 interaction and inhibiting membrane fusion of the viral envelope. Based on in vitro data against SARS renewed attention in the treatment of COVID-19. ${ }^{12}$

\section{Remdesivir}

Remdesivir is an intravenous (IV) investigational nucleotide prodrug of an adenosine analogue. Remdesivir binds to the viral enzyme RNA-dependent RNA polymerase. The host metabolizes Remdesivir into active NTP, which competes with ATP for incorporation into the nascent RNA strand, resulting in premature termination of RNA synthesis, halting the growth of the RNA strand. ${ }^{13}$ 


\section{IMMUNOMODULATORS}

\section{Glucocorticoids}

Low doses of methylprednisolone have the potential to the prevention of extended cytokine response and may accelerate the resolution of pulmonary and systemic inflammation in pneumonia. It is believed that methylprednisolone, may improve dysregulated immune response caused by sepsis (a possible complication of infection with COVID-19) and increase blood pressure when it is low. ${ }^{11}$ RECOVERY, a multicentre, randomized, open-label trial in hospitalized patients with COVID-19, showed that the mortality rate was lower among patients who were randomized to receive dexamethasone than among those who received standard of care. ${ }^{14}$

\section{Interferon}

IFNs- $\alpha / \beta$ are broad-spectrum antivirals, exhibiting both direct inhibitory effects on viral replication and indirect supporting an immune response to clear virus infection. IFN treatment accelerated viral clearance and reductions in circulating IL- 6 and CRP levels. The advantage of IFN- $\alpha 2 b$ over blocking IL-6 rests in IFN targeting the cause (SARSCoV-2), not only the symptoms (IL-6). ${ }^{12}$

\section{Interleukin 6 antagonists}

Syndrome of dysregulated and systemic immune overactivation described as a cytokine storm or hyperinflammatory syndrome worsens acute respiratory distress syndrome in hospitalised COVID 19 patients. The surge in IL-6 along with viral load is perceived in severe cases. By inhibiting IL-1 signalling, decline NF- $\kappa$ B-mediated upregulation of multiple cytokines, including IL-6.Early immunomodulatory intervention can prevent the demand for mechanical ventilation..$^{15}$ Tocilizumab-recombinant humanized anti-IL6R monoclonal antibody. FDA approved for rheumatologic disorders. CORIMUNO-TOCI trial is in pipeline.

\section{Kinase inhibitors}

These drugs prevent phosphorylation of key proteins involved in the signal transduction that leads to immune activation and inflammation. The inflammation and associated immunopathologies observed in patients with COVID-19 can be reduced by the immunosuppressive action of kinase inhibitors.

\section{Acalabrutinib}

Acalabrutinib is a second-generation, oral Bruton tyrosine kinase inhibitor that can be proposed for COVID-19 because it can modulate signalling that promotes inflammation. ${ }^{16}$

\section{Ibrutinib}

Ibrutinib is a first-generation Bruton tyrosine kinase inhibitor. The conjecture of Ibrutinib in improving inflammation and protection against lung injury in patients with COVID-19 can also be considered as a target. ${ }^{17}$

\section{Zanubrutinib}

Zanubrutinib is a second-generation, oral Bruton tyrosine kinase inhibitor proposed to be of use in patients with COVID-19 by modulating signalling that promotes inflammation. $^{18}$

\section{Baricitinib}

Baricitinib is an oral Janus kinase inhibitor that is selective for JAK1 and JAK2, can modulate downstream inflammatory responses via inhibition of JAK1/JAK2 kinase and has exhibited a dose-dependent inhibition of IL-6-induced STAT3 phosphorylation. ${ }^{19}$

\section{Ruxolitinib}

Ruxolitinib is an oral Janus kinase inhibitor selective for JAK1 and JAK2 and is theorized to have antiviral properties through inhibition of AAK1, which may prevent viral entry and infection of pulmonary AT2 epithelial cells. ${ }^{20}$

\section{CoVID-19 convalescent plasma}

Plasma from donors who recovered from COVID-19 may contain antibodies. SARS-CoV-2that may help suppress the virus and modify the inflammatory response. ${ }^{21}$ The management of COVID-19 with plasma therapy involves the process of plasma retrieval rich in virus-neutralizing antibodies from the recovered antibodies and transfusing them to COVID-19 positive patients.

\section{Ivermectin}

In the cytoplasm, Importin $\alpha / \beta 1$ and coronavirus cargo protein bind each other and translocates through the nuclear pore complex (NPC) into the nucleus where the complex break down and the host cell's antiviral response is increased leading to enhanced infection. Ivermectin binds to and destabilises the Imp $\alpha / \beta 1$ heterodimer thereby preventing Importin $\alpha / \beta 1$ from binding to the viral protein and preventing it from entering the nucleus. This likely results in reduced inhibition of the antiviral responses, leading to a normal, more efficient antiviral response. ${ }^{22}$

\section{Anticoagulants}

Coagulative and fibrinolytic abnormalities are closely involved in the COVID-19 pathogenesis and have a big effect on prognosis. non-vitamin $\mathrm{K}$ antagonist oral anticoagulants (NOACs) can present side-effects caused by extensive interactions with the drugs currently in use for the therapy of patients with COVID-19, whereas low-molecular-weight heparins do not exhibit significant interactions; thus, the latter is currently the first-choice drug to prevent thromboembolic phenomena in these patients. ${ }^{23}$ 


\section{DEVELOPMENT OF IMMUNITY- HOPE TO BE A SOLUTION}

\section{What is immunity?}

Immunity can be defined as a complex biological system endowed with the capacity to recognize and tolerate whatever belongs to the self and to recognize and what is foreign (nonself). Biological immunity can refer to constitutive physical innate mechanisms, such as the activity of natural killer (NK) cells against virus-infected cells. Immunity can also be innate but inducible, as in an antiviral state induced by exposure to double-stranded (dsRNA). ${ }^{24}$ There are two types of acquired immunity: active and passive..$^{25}$
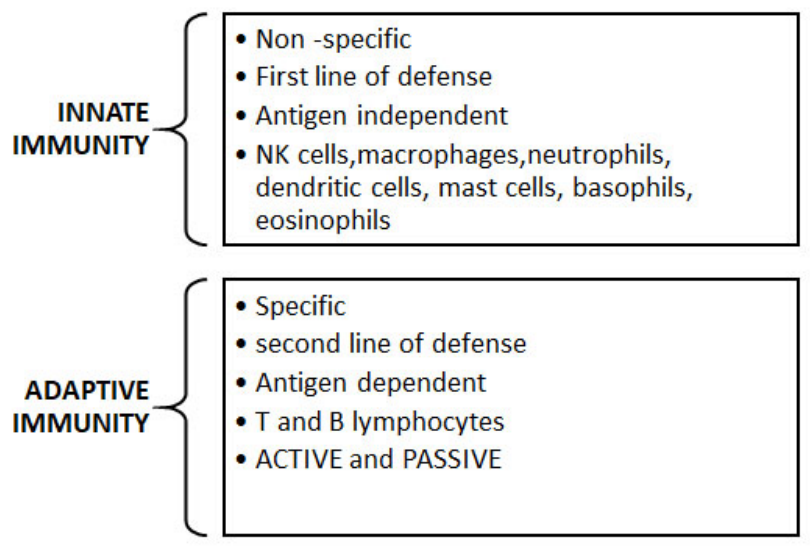

Figure 1: Differences between Innate \& Adaptive Immunity

\section{Active Immunity}

Active immunity results when exposure to a pathogen triggers the immune system to produce antibodies to that disease. This exposure to the pathogens or toxins can occur through infection with the disease or institution of a killed or weakened form of the pathogens, its toxins or its surface proteins through vaccination. Nevertheless, when an immune person comes into contact with that same disease succeedingly, their immune system will recognize it and immediately produce the antibodies needed to fight it. Active immunity is longlasting and sometimes life-long.

\section{Passive Immunity}

Immunity is said to be passive when a person is given antibodies to a disease rather than producing them through his or her immune system.

A newborn baby acquires immunity from its mother through the placenta. Antibody-containing blood products such as immunoglobulin can right away impart immunity in an individual. The major advantage of passive immunity is protection is immediate whereas active immunity takes time. Immune response to SARS-CoV-2 involves both cell-mediated immunity and antibody protection.

\section{TRADITIONAL VS. PANDEMIC VACCINE DE- VELOPMENT}

Traditional vaccine development shows in upper while that in the pandemic condition is depicted in the lower panel.
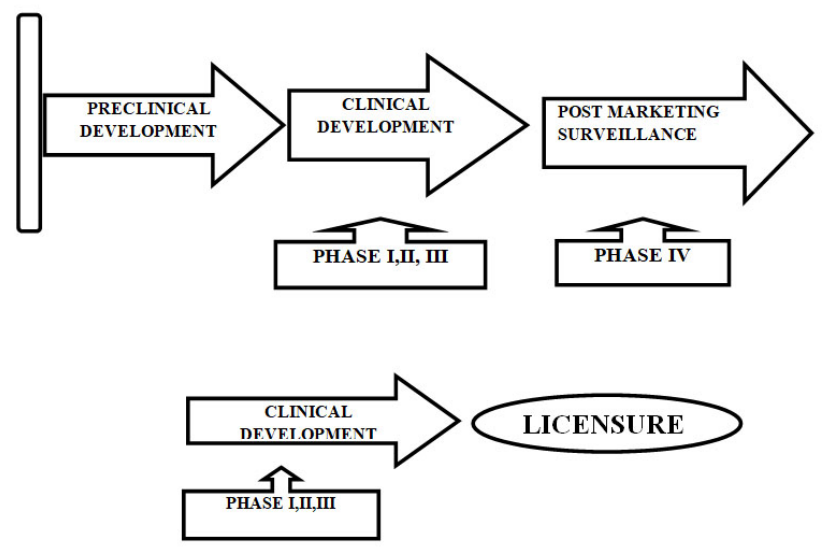

Figure 2: Difference between conventional and pandemic vaccine development.

\section{COVID 19 VACCINATION IMPORTANCES}

The current danger of avian-flu to the human population globally, the potential for the reappearance of serious, intense respiratory disorder (SARS) - related Coronavirus and the distinguishing proof of numerous novel respiratory infections underline the need for the improvement of remedial and preventive procedures to battle the viral disease..$^{26}$ Antibody improvement is a key element in anticipation of broad viral contamination and the decrease of mortality related to viral diseases.

Previously infected animal models such as mice and hamsters are protected from subsequent infection with SARSCo V in the absence of enhanced disease and vaccine studies, and passive immunoprophylaxis performed with these animals suggest that previous exposure and the presence of Neutralizing antibodies provide protection. ${ }^{27}$

Coronavirus vaccines can be of various types:

1. Inactivated Coronavirus vaccines

2. Live attenuated Coronavirus vaccines

3. S protein-based vaccines

4. Vectored vaccines

5. DNA Vaccine

\section{CONSIDERATIONS IN COVID -19 VACCINE DEVEL- OPMENTS}

- Vaccine development requires Biosafety level 3 precautions.

- Incomplete inactivation can be a potential health threat. 
- Live attenuated vaccine strain may also be shed in faeces, with chances of spreading of unvaccinated individuals.

- Vaccines targeting $\mathrm{S}$ protein could induce antibodies that can block virus binding

- Can also be given in combination.

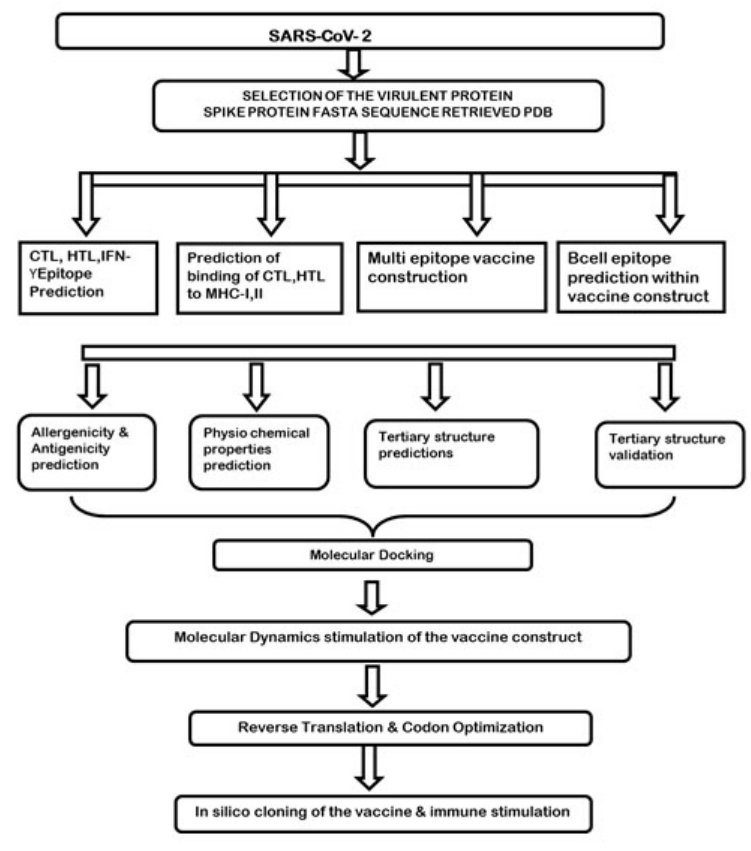

Figure 3: Process of Vaccine Development.

\section{BCG vaccine hope in COVID-19}

An interesting hypothesis suggests that multiple doses of tuberculosis vaccine bacillus Calmette-Guérin (BCG) could be protective against COVID-19 due to the so-called trained immunity phenomenon. A recent preprint revealed nonspecific beneficial effects of universal BCG vaccination on hindering COVID-19 spread and its complications in some countries (e.g., Japan). ${ }^{28}$

BCG-CORONA (Reducing Health Care Workers Absenteeism in COVID-19 Pandemic Through BCG Vaccine) trial ${ }^{29}$ and BRACE trial (BCG Vaccination to Protect Healthcare Workers Against COVID-19. ${ }^{30}$ The nonspecific protective effect of the BCG vaccine can be clarified only when results of the above two ongoing trials become available.

\section{Measles, Mumps, Rubella (MMR) Vaccine hope in COVID-19}

There is a recent past report of reduction of under-five mortality with the increased uptake of the MMR vaccine. The interferons elicited by the vaccine are found to be more effective as well as the NK cells induced by vaccines showing robust antiviral property. ${ }^{31}$ Thus, coupled with the advantage of vaccine-induced IFNs, NK cells and the cross-protective antibodies, successfully can ameliorate SARS-CoV-2 infection.

The common properties between MMR Viruses \& SARS$\mathrm{COV}$ in terms of transmission and their primary replication in the upper respiratory tract and possible cross-protective innate immunity offered by vaccination is prompting us to suggest the repurposing of MMR vaccine for COVID-19. ${ }^{32}$

\section{Benefits of vaccination in arresting the cy- tokine storm}

The Pathogenesis involved in severity and mortality associated with COVID-19 cases is thought to be tied to the cytokine storm that includes the production of the pro-inflammatory cytokines (IL-1 $\beta$, IL-6, IL-12, IL-18, IL-33, TNF- $\alpha$, TGF- $\beta$ ) that causes IFN and NK cell production. Experimental studies are showing that vaccination followed by reinfection stimulates only very low levels of cytokine production, which may result in mild infection. ${ }^{33}$ This might explain the mild SARS-CoV-2 infections in children globally who had recent vaccination particularly viral.

\section{COVID VACCINATION ECHELON}

There are progressing clinical trials for the development of prophylactic vaccines globally. There are various molecular platforms like non replicating viral vector, RNA-based vaccine, Protein subunit, DNA-Based, inactivated virus, live attenuated vaccine etc. As the status of September 2, 2020, 218 vaccines are undergoing various stages of clinical trials. ${ }^{34}$

\section{COVID VACCINE TRIAL STATUS IN INDIA}

\section{COVAXIN by Bharat Biotech}

Bharat Biotech, an Indian biotechnology company in Hyderabad, is partnering with the National Institute of Virology (NIV) of INDIAN COUNCIL OF MEDICAL RESEARCH (ICMR) to develop an inactivated vaccine candidate( $\mathrm{BBV}$ 152) for COVID-19 called Covaxin. A Phase $1 / 2$ trial of about 1,100 healthy participants is under process after approval by the Drug Controller General of India. ${ }^{35}$ In addition to Covaxin, Bharat Biotech is also working on two other vaccine candidates: one( BBV 150) with the University of Wisconsin-Madison and FluGen which is an intranasal vaccine based on an influenza virus where gene sequences from SARS-CoV-2 are inserted into M2SR- vaccine platform(M2ion channel protein Deficient single replication ) which completed Phase II trials in the USA. ${ }^{36}$ And the other ( BBV 151) with Thomas Jefferson University.

Indian Council of Medical Research has selected 12 institutes apart from one in Odisha for a clinical trial of the country's first COVID-19 vaccine coaxing. The 12 institutes are from Vishakhapatnam, Rohtak, NewDelhi, Patna, 
Belgaum(Karnataka), Nagpur, Gorakhpur, Tamilnadu, Hyderabad, Arya Nagar, Kanpur \& Goa. ${ }^{37}$

The trial's principal investigator reported that the early results in the first 50 people who received the vaccine candidate appear to be "encouraging,". There is hope of vaccine in the market by the year-end of $2020 .^{38}$

\section{ZyCoV-D BY ZYDUS CADILA}

India's Zydus Cadila is developing two vaccine candidates. The first ZyCoV-D, a plasmid DNA vaccine candidate for COVID-19 that targets the viral entry membrane protein of the virus. On 5 August 2020, Zydus announced the completion of Phase 1 and the candidate was entering Phase 2. The second approach deals with a live attenuated recombinant measles virus vectored vaccine. ${ }^{39}$

\section{ChAdOx1nCoV-19 by SERUM INSTITUTE OF INDIA:}

Immunological drugs manufacturer developing two vaccines. The first, which is a Live attenuated virus candidate in partnership with Codagenix and the second one the measles based viral vectored vaccine, with Themis. At present, both vaccines are in the pre-clinical stage. Also, SIIPL developing VPM1002 which is an immune enhancer. Trials were initiated in India, Germany and will start shortly in Canada, Australia and New Zealand. In India, now it is in phase III. ${ }^{40}$ As of now, the vaccine developed by Oxford university Jenner institute might the first Coronavirus vaccine to be available for Indians by the end of $2020 .^{40}$

\section{Vaccine by Indian Immunologicals, India}

One of the prime vaccine makers in Hyderabad, India, Developing a Live attenuated vaccine in collaboration with Australia's Griffith University using the latest codon de-optimization technology and they are planning mass production of the vaccine candidate with Vero cell platform technology. ${ }^{41}$

\section{Vaccine of Biological $E$}

The most recent entrant in the vaccine race developing the Recombinant protein subunit, an adjuvant subunit vaccine comprising the Receptor Binding Domain (RBD) of SARS$\mathrm{CoV}-2$ spike protein as the vaccine candidate which completed pre-clinical studies. ${ }^{42}$

\section{Vaccine of Mynvax}

Bengaluru based medical pharmaceutical startup, a few months back announced their work on COVID-19 Vaccine which is protein-based, in collaboration with the Indian Institute of Science and the vaccine candidate now in preclinical stage. ${ }^{43}$

\section{Vaccine of Panacea Biotec}

Delhi based biotechnology company, in partnership with RefanaInc., a US-based pharma company to develop, manufacture \& distribute inactivated vaccine strain for COVID-19. The vaccine candidate in preclinical development at present and expected to start the human trials by October $2020 .{ }^{43}$

\section{CONCLUSION}

Globally many countries are affected by the rapid outbreak of SARS-CoV-2 so we are in urgency to develop a safe and effective vaccine. At present, there are about 2400 ongoing clinical trials globally including more than 200 clinical trials in India with various therapeutic interventions including vaccines and repurposing of existing drugs. Vaccine development is based on the knowledge gained from SARS \& MERS vaccine development strategies. The receptor-binding domain of $\mathrm{S}$ protein is considered as the main target for developing COVID vaccine candidates, as shown in several preclinical studies. At present, the various biopharmaceutical companies as well as academic sectors are in a race to develop the COVID-19 vaccine by several platforms. To make an effective SAR-CoV-2 vaccine possible, there are many parameters to be considered like target identification, immunization route, immune protection, suitable animal models, scalability, production facility target population etc. Hence it may take still more time because of the limitations such as the unavailability of appropriate animal models to check efficacy, immunogenicity and toxicity of the vaccine before the clinical trials in human. So there is a need for coordination and technology transfer among governments, regulatory agencies, pharmaceutical companies, and the World Health Organization (WHO), for the global production of an effective vaccine against SARS-CoV-2. As well as once the vaccine is on the market the next challenge will be the affordability and availability of vaccines. India is one of the pharmacological therapeutics distributors globally, there should be enough resources for the production and the distribution of vaccines to other countries as well. Further, the evaluation of immunity and long-term memory of COVID-19 recovered individuals may be helpful in the development of a potential prophylactic and therapeutic agent not only against SARS-CoV-2 but other similar coronaviruses during a future outbreak.

\section{ACKNOWLEDGEMENT}

We the authors are thankful to all faculty, our seniors and co pg's for their help and support.

\section{Conflict of Interest}

Nil economic interest or conflict exists for the authors. 


\section{Financial Support}

Nil funding sources.

\section{REFERENCES}

1. Lu H, Stratton C, Tang Y. Outbreak of pneumonia of unknown aetiology in Wuhan, China: the mystery and the miracle. J Med Virol 2020;92:401-402.

2. WHO. Coronavirus Disease 2019 (COVID-19) Situation Report-99; 2020.

3. Li LQ, Huang T, Wang YQ. COVID-19 patients' clinical characteristics, discharge rate, and fatality rate of meta-analysis. J Med Virol 2020;92(6):577-583.

4. Hashem AM, Alghamdi BS, Algaissi AA, Alshehri FS, Bukhari A, Alfaleh MA, et al. Therapeutic use of chloroquine and hydroxychloroquine in COVID-19 and other viral infections: A narrative review. Travel Med Infect Dis 2020;35:101735.

5. Mehta P, McAuley DF, Brown M, Sanchez E, Tattersall RS, Manson JJ. COVID-19: Consider cytokine storm syndromes and immunosuppression. Lancet 2020;395:1033-1034.

6. Meng J, Xiao G, Zhang J. Renin-angiotensin system inhibitors improve the clinical outcomes of COVID-19 patients with hypertension. Emerg Microbes Infect 2020;9(1):757-760.

7. Ye R, Liu Z. ACE2 exhibits protective effects against LPSinduced acute lung injury in mice by inhibiting the LPS-TLR4 pathway. Exp Mol Pathol. 2020; 113:104350.

8. Sanders JM, Monogue ML, Jodlowski TZ, Cutrell JB. Pharmacologic Treatments for Coronavirus Disease 2019 (COVID-19): A review. JAMA 2020; 323(18):1824-1836.

9. De Meyer S, Bojkova D, Cinatl J, Damme EV, Buyck C, Loock $\mathrm{MV}$, et al. Lack of antiviral activity of darunavir against SARSCoV-2. Int J Infect Dis 2020; 97:7-10.

10. Finkelman-Rodrigues N, Sacramento CQ, Lima CR, da Silva FS, Ferreira AC, Mattos M, et al. Atazanavir, Alone or in Combination with Ritonavir, Inhibits SARS-CoV-2 Replication and Proinflammatory Cytokine Production. Antimicrob Agents Chemother 2020;64(10):e00825-20.

11. Furuta Y, Komeno T, Nakamura T. Favipiravir (T-705), a broadspectrum inhibitor of viral RNA polymerase. Proc Jpn Acad Ser B Phys Biol Sci 2017;93(7):449-463.

12. Blaising A, Polyak SJ, Pécheur E-I. Arbidol as a broad spectrum antiviral: an update. Antiviral Res 2014;107:84-94.

13. Eastman RT, Roth JS, Brimacombe KR . Remdesivir: A Review of Its Discovery and Development Leading to Emergency Use Authorization for Treatment of COVID-19. ACS Cent Sci 2020;6(5):672-683.

14. The RECOVERY Collaborative Group. Dexamethasone in Hospitalized Patients with Covid-19, Preliminary report. N Engl J Med 2021; 384:693-704.

15. Ingraham NE, Lotfi-Emran $\mathrm{S}$, Thielen BK, Techar K, Morris RS, Holtan SG, et al. Immunomodulation in COVID-19. Lancet Resp Med 2020;8(6):544-546

16. Owen C, Berinstein NL, Christofides A, Sehn LH. Review of Bruton tyrosine kinase inhibitors for the treatment of relapsed or refractory mantle cell lymphoma. Curr Oncol 2019;26(2):e233e240.

17. Treon SP, Castillo JJ, Skarbnik AP. The BTK inhibitor ibrutinib may protect against pulmonary injury in COVID-19-infected patients. Blood 2020;135(21):1912-1915.

18. Zanubrutinib (Brukinsa) [package insert]. Food and Drug Administration. November 2019.
19. McInnes IB, Byers NL, Higgs RE, Comparison of baricitinib, upadacitinib, and tofacitinib mediated regulation of cytokine signalling in human leukocyte subpopulations. Arthritis Res Ther 2019;21(1):183.

20. Stebbing J, Phelan A, Griffin I. COVID-19: combining antiviral and anti-inflammatory treatments. Lancet Infect Dis 2020;20(4):400-402.

21. Wang X, Guo X, Xin Q, Pan Y, Hu Y, Li J, et al. Neutralizing antibodies responses to SARS-CoV-2 in COVID-19 inpatients and convalescent patients. Clin Infect Dis 2020;71(10):2688-2694.

22. Carly L, Druce JD, Catton MG, Jans DA, Wagstaff KM. The FDA-approved drug ivermectin inhibits the replication of SARS-CoV-2 in vitro. Antiviral Res 2020;178:104787.

23. Wang D, Hu B, Hu C. Clinical characteristics of 138 hospitalized patients with 2019 novel coronavirus-infected pneumonia in Wuhan, China. J Ame Med Ass. 2020;323:1061-1069.

24. Rodgers JR. In Encyclopedia of Microbiology ( ${ }^{\text {rd }}$ edition), 2009.

25. Delves PJ. Acquired immunity review. MSD Manual consumer Version, Apr 2020.

26. Ankur C, Manushree P. COVID-19: a systematic review on corona virus. Int J Pharmacol 2020;7(4):96-100.

27. Beck MA, Matthews CC. Micronutrients and host resistance to viral infection. Proc Nut Soc 2000;59:581-585.

28. Sala G, Miyakawa T. Association of BCG vaccination policy with prevalence and mortality of COVID-19. medRxiv preprint doi: https://doi.org/10.1101/2020.03.30.20048165

29. ClinicalTrials.gov. Reducing health care workers absenteeism in COVID-19 pandemic through BCG vaccine (BCG-CORONA). https://clinicaltrials.gov/ct2/show/NCT04328441. Updated 2020.

30. ClinicalTrials.gov. BCG vaccination to protect healthcare workers against COVID-19 (BRACE). https://clinicaltrials.gov/ct2/ show/NCT04327206. Updated 2020.

31. Moss WJ, Scott S. WHO immunological basis for immunization series module: measles. World Health Organization: 2008 Aug. https://www.who.int/immunization/sage/Module_on_Measles_ Immunology_26Aug08.pdf

32. Anbarasu A, Ramaiah S, Livingstone P. Vaccine repurposing approach for preventing COVID 19: can MMR vaccines reduce morbidity and mortality? Human Vaccines Immunotherap 2020;16(9): 2217-2218.

33. Cameron MJ, Kelvin AA, Leon AJ, Cameron CM, Ran L, Xu L, et al. Lack of innate interferon responses during SARS coronavirus infection in a vaccination and reinfection ferret model. PLoS One. 2012;7(9):e45842.

34. Vaccine Tracker- Regulatory Affairs Professional Society. COVID-19 vaccine tracker $\mid$ RAPS.

35. Bharat Biotech's Covaxin vaccine yields positive Phase I data; CLINICAL TRIALS AReNA; 17 August 2020

36. COVID-19 Coronavirus Pandemic cases, Worldometer. Available at https://www.worldometers.info/coronavirus

37. ICMR selects 12 institutes for the clinical trial of COVID-19 vaccine: Healthworld.com From The Economic Times July 03, 2020.

38. Coronavirus vaccine update; times of india.com August 21, 2020

39. Zydus Cadila launches a fast-tracked programme to develop a vaccine for the novel coronavirus, 2019-to(COVID-19). Available online: https://pipelinereview.com

40. Codagenix and Serum Institute of India Initiate Co-Development of a Scalable, Live-Attenuated VaccineAgainst the 2019 Novel Coronavirus, COVID-19. https://www.biospace.com/ article/codagenix-and-serum-institute-of-india-initiate-co-de- 
velopment-of-a-scalable-live-attenuated-vaccine against-the2019-novel-coronavirus-covid-19

41. Ewen $C$. The race for coronavirus vaccines: a graphical guide. Nature 2020;580(7805):576-7.

42. World Health Organization Director-General's opening remarks at the media briefing on COVID-19 - 11 March 2020. Avail- able at https://www.who.int/dg/speeches/detail/who-directorgeneral-s-opening-remarks-at-the-media briefing-on-covid19-11-march-2020

43. Coronavirus vaccine Development In India: Here's A List Of All Vaccines Being Locally Made \& Developed: Times of India: July 20, 2020 